\title{
Mapping Indoor Environments Based on Human Activity
}

Slawomir Grzonka

Frederic Dijoux

Abstract - We present a novel approach to build approximate maps of structured environments utilizing human motion and activity. Our approach uses data recorded with a data suit which is equipped with several IMUs to detect movements of a person and door opening and closing events. In our approach we interpret the movements as motion constraints and door handling events as landmark detections in a graph-based SLAM framework. As we cannot distinguish between individual doors, we employ a multi-hypothesis approach on top of the SLAM system to deal with the high data-association uncertainty. As a result, our approach is able to accurately and robustly recover the trajectory of the person. We additionally take advantage of the fact that people traverse free space and that doors separate rooms to recover the geometric structure of the environment after the graph optimization. We evaluate our approach in several experiments carried out with different users and in environments of different types.

\section{INTRODUCTION}

The problem of localizing and tracking people has recently received substantial attention in the robotics community as knowledge about the current position of a person and his or her activity allows a mobile robot to improve its services to its users. For example, the robot can better anticipate future actions of the person. Additionally, knowledge about the environment and the location of people can greatly support search and rescue missions in emergency situations. Consider, for example, firefighters in a building enclosed by smoke and fire. If a map of the environment can be constructed while the firefighters are within the building, an operator or automated system can re-route the people to the exit in case of an emergency. Alternatively, one could use the map of the environment to more intelligently coordinate the actions of the rescue workers to more effectively search the environment for potential victims and at the same time reduce the time the rescue workers are exposed to potential threats.

In this paper, we consider the problem of simultaneously estimating the trajectory of a person walking through an indoor environment and the map of the environment. Our approach utilizes the movements of a person as well as door handling activities to reconstruct the trajectory of the person as well as the map of the environment. The estimation is carried out based on data recorded with an Xsens data suit, which is equipped with 17 inertial measurement units (IMUs), worn by a human. This data suit records full body postures of the person and in this way allows for a prediction of the motion as well as for the identification of unique and

Department of Computer Science, University of Freiburg, D-79110 Freiburg, Germany

\author{
Andreas Karwath \\ Wolfram Burgard
}
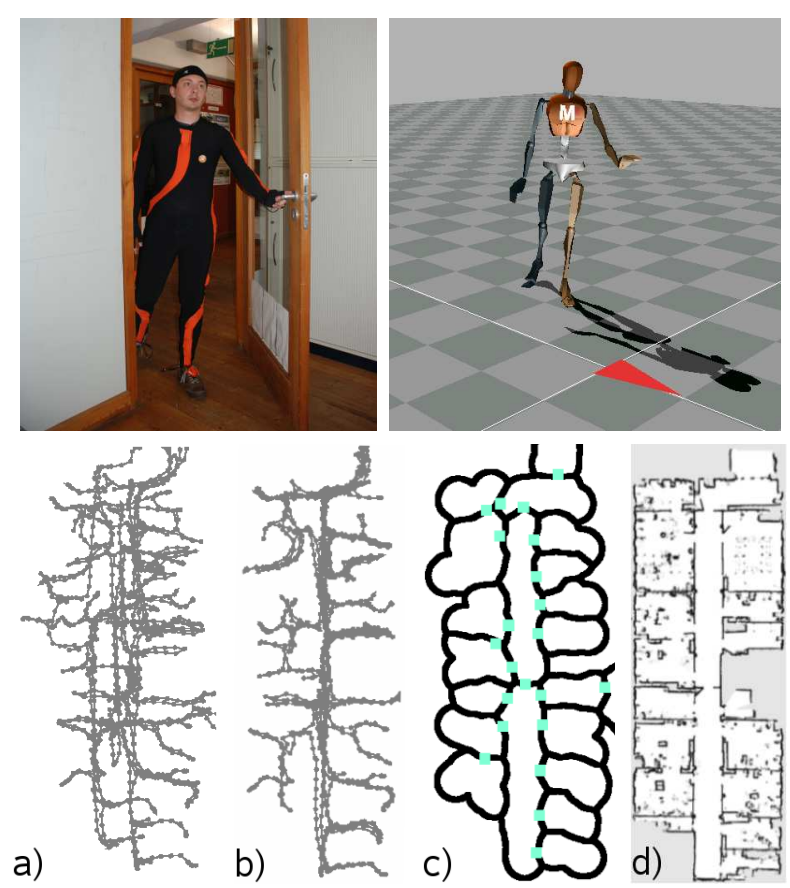

Fig. 1. Human activities like opening a door (top left and right) are used to detect loop closures. Given this information, the pure odometry (a) can be corrected (b) leading to a consistent trajectory. Based on this corrected odometry and the knowledge about the location of individual doors, an approximate map of the environment can be calculated (c). Here, light blue/gray squares indicate the location of individual doors. A laser based map of the same environment is shown in (d) for comparison.

location-based activities such as door handling events. The two top images of Figure 1 depict a person wearing the Xsens data suit (left) and the posture estimated by the software delivered with it (right).

We present an approach that is able to learn the motions carried out by a human during handling a door with either the left or the right hand. This learned motion is then used to detect door handling events and at the same time to estimate the location of doors while the human is walking through the environment. We then apply a graph-based SLAM approach that uses the odometry estimated by the IMUs and the landmarks corresponding to the door handling events to estimate the true path of the person. To deal with the corresponding data association uncertainty in the landmark determination, we apply a multi-hypothesis scheme. After calculating the path of the person, we utilize the pose of the estimated doors to calculate an approximate two-dimensional map of the environment.

The paper is structured as follows. After discussing related work we briefly describe the algorithms used for learning and detecting the motion for handling a door. Section IV re- 
views the multi-hypothesis tracker for sensors providing only positive feedback, especially the expressions to calculate the hypothesis probabilities. Section V describes how we detect potential loop closure candidates and our overall system. In Section VI we present our experimental results based on real data recorded by different people walking inside and outside of various buildings.

\section{RELATED WORK}

The problem of human indoor navigation and localization has recently become an active research field [6], [12], [7], [2]. A number of different sensors are employed as well as different kind of localization techniques were used. One of the first works in this area is the one by Lee and Mase [6], where wearable accelerometers and other sensors, like a digital compass and a velocity sensor, were employed to recognize when humans perform specific activities and switch between indoor locations. They integrate the accelerometer data over time to localize humans within a known environment, using higher level descriptors like standing - 2 steps north - 40 steps east - etc. The field of indoor navigation and localization is therefore closely related to activity recognition using accelerometer data. [1], [10] present approaches to predict certain low level activities like walking , standing, running, sit-ups, and others using purely extracted features from raw accelerometer data and a variety of different learning algorithms. However, they do not employ this information for indoor positioning. In [12], the authors utilize an accelerometer together with an infrared proximity sensor mounted on a pair of headphones to detect when a human is passing through a doorway. In this work, the authors are able to construct topological maps, where rooms are represented by single nodes and edges represent the path in steps between doorways. For building these maps and for detecting loop closures, the human user has to indicate by gesture which door was passed, i.e. giving each door a unique identifier via the infrared proximity sensor. Within this map, the approach also allows for localization based on Bayesian filtering. HeadSLAM by Cinaz and Kenn [2] employs a laser scanner together with an IMU mounted on a helmet. They use the IMU sensor to be able to project the laser scans into a horizontal plane in a global coordinate system and employ a modified GMapping [5] implementation, by incorporating a simple motion model with either a fixed speed assumption for walking or no speed while standing.

\section{Motion TEMPlates}

Since beside the current pose of the body segments no further information is available, we need to track those motions in order to detect activities like opening/closing a door. Without this additional information we can not detect loop closures and we can only generate an approximate map based on the current odometry. This, however, would lead to an inconsistent map due to small errors accumulating over time, as shown in Figure 1(a). We therefore propose to detect the motion used for handling a door based on motion templates $(M T)$ as proposed by Müller et al. [8]. a)

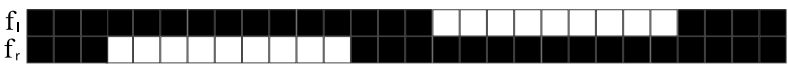

b)

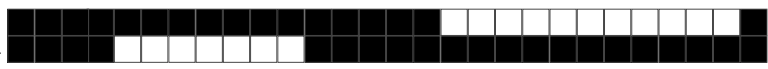

c)

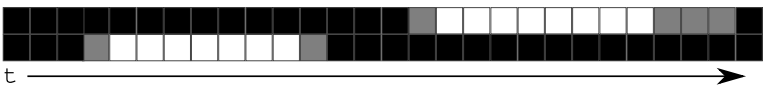

Fig. 2. A synthetic example: Given two examples (a) and (b) of the same motion walking. The features $f_{l}, f_{r}$ are 1 (white) iff the left/right foot is in front of the body and 0 otherwise. The resulting merged template is depicted in (c). Here, gray areas indicate the value 0.5 , meaning don't care. Intuitively, the matrix can be interpreted through the following sections: feet parallel, right foot in front, feet parallel, left foot in front, feet parallel.

The key idea of the work by Müller et al. is to use simple boolean features like right hand is above head and create more expressive features (motion templates) by conjunction of the simple ones. Given $f$ of those features and a motion sequence of length $K$ this leads to a matrix of size $f \times K$. Note that each entry of this matrix is either 1 or 0 indicating this feature being active or not at the specific time and that the sequence length $K$ can in general be different for each motion sequence. Consider for example the two features $f_{l}, f_{r}$ with $f_{l}$ indicating the left foot being in front of the body and $f_{r}$ active if the right foot is in front of the body. Given this set of features, a typical walking template for two different sequences of the same length could look like Figure 2(a) and (b). The learned template given these two examples is depicted in Figure 2(c). Here, black and white correspond to 0 and 1 respectively. The grayshaded boxes account for the fact 0.5 meaning don't care. More formally, let $C$ denote a motion class consisting of $s$ templates $\mathrm{MT}_{1} \ldots \mathrm{MT}_{s}$ indicating the same motion. Each template $\mathrm{MT}_{j}$ is described by a matrix of size $f \times K_{j}$ and a weight vector $\alpha_{j}$ with $\alpha_{j}(k)$ being the weight of the $k$-th column. Initially, we set all weights to 1 indicating each time step to be equally important. The learning of a class reference template CT is done performing the following steps. First, we select a template $\mathrm{MT}_{i}$ from the class $C$ and use it as a reference $R$. We then compute an optimal alignment of this reference template to all other templates from the same class utilizing Dynamic Time Warping (DTW) [9]. We alter the current motion template $\mathrm{MT}_{j}$ (which was aligned to $R$ ) in the following way. Given $n$ columns of the reference template $R$, namely $R(k), \ldots, R(k+n-1)$, are matched to one column of the current template $\mathrm{MT}_{j}(l)$, this column $\operatorname{MT}_{j}(l)$ is duplicated $n$ times having the new weight $\alpha(l) / n$. In case one column $R(k)$ is matched to $m$ columns of $\mathrm{MT}_{j}$, namely $\operatorname{MT}_{j}(l), \ldots, \operatorname{MT}_{j}(l+m-1)$, these $m$ columns are averaged (multiplied by their weights) to one with the new weight being the sum of these columns' weights. This process is called warping. Now, that each $\mathrm{MT}_{j}$ has the same length as the reference $R$, we calculate in the second step a weighted average template from all templates based on the columns and their associated weights. Consequently, this step is called averaging. In the third step, called unwarping we stretch and contract the resulting template so that all but eventually the last column have a weight of 1 . In this case, given the weight of a column $\alpha(k)<1$, we merge this column with 
subsequent ones until the weight is 1 . In case the weight of a column $\alpha(k)>1$, we duplicate this column into two columns, one with weight 1 and the second with weight $\alpha(k)-1$. The process is then repeated for the next column.

We repeat the whole procedure of warping, averaging, and unwarping for all templates $\mathrm{MT}_{i}$ where every template once is the reference template $R$. After this step, we replace all templates by the outcome of the procedure when this template was the reference $R$. Since the selection of the reference $R$ induces a bias on the learned template, we iterate the whole procedure until no major change between the different templates exists. The outcome of this algorithm is the class template CT. Note that due to the steps warping, averaging and unwarping the values of the resulting matrix are now $\in[0,1]$ instead of $\in\{0,1\}$. The final step therefore consists of changing each entry of CT into either 0,1 or 0.5 , with 0.5 indicating the flag don't care. We achieve this by selecting a threshold $\gamma$ and changing each entry to 0 , given its current value $<\gamma$. The value is altered to 1 if it was $>(1-\gamma)$ and set to 0.5 otherwise. In all our experiments we set $\gamma$ to 0.1 . An example of such a template is depicted in Figure 2(c).

Now, given the learned class template CT and a new motion sequence, we can calculate a similarity between both. Therefore, we align the motion template of the actual sequence to CT via DTW. We obtain a score between both templates by dividing the amount of mismatches by the number of used cells (i.e., the cells being either 0 or 1). Given this score is below a threshold $\tau$, the actual motion sequence is said to belong to the motion class CT.

Since we are only interested in the motion used for handling a door with either the left or the right hand we use features based on the pose and velocity of the hands only. Intuitively, we use a set of features describing whether the hand is at the level of the door handle, whether it is raising, hold still or lowered, and finally whether the hand is moving towards the body or away from it. We learned the template for the activity handling a door, which consists of the four subclasses open left, close left, open right, close right, using 10 examples from a training data set for each subclass. Based on a second validation data set, we selected the threshold $\tau=0.25$ for detecting the motion. Using this threshold, we did not encounter false positives on the validation data set. Although the features used for detecting a door are quite simple, we can reliably detect the timestamp when the door handle was touched within 1.5 seconds of the true timestamp (i.e., manually labeled ground truth). Therefore, we can now use the pose of the hand as an approximation of the location of the door. Given this algorithm we are able to detect when we touched $a$ door, but not which one. We therefore have to take care about possible data associations, which is described in the next section.

\section{Multi Hypothesis Tracking}

In this section we review the Multi Hypothesis Tracker (MHT) as described by Reid [11] for sensors providing only positive feedback. In the original paper by Reid, this type of sensor is called a "type 2" sensor. There, any measurement can be either detected (assigned to an existing track), marked as a false alarm or as a new track. Since in our particular case the tracks are static doors, we will call them doors in the remainder of this section, rather than tracks. As described in Section III we select a threshold for detection in such a way, that we do not have to model false positives. Therefore, a measurement can only be interpreted as detected (when matched to an existing door) or as a new door. In order to derive the probabilities of individual measurement assignments we briefly reconsider the formulation of the Multi Hypothesis Tracker for type 2 sensors.

Let $\Omega_{j}^{k}$ be the $j$-th hypothesis at time $k$ and $\Omega_{p(j)}^{k-1}$ the parent hypothesis from which $\Omega_{j}^{k}$ was derived. Let further $\Psi_{j}(k)$ denote an assignment, that based on the parent hypothesis $\Omega_{p(j)}^{k-1}$ and the current measurement $z_{k}$ gives rise to $\Omega_{j}^{k}$. The assignment set $\Psi_{j}(k)$ associates the current measurement either to an existing door or a new door. Given the probability of an assignment and the probability of the parent hypothesis $\Omega_{p(k)}^{k-1}$, we can calculate the probability of each child that has been created through $\Psi_{j}(k)$. This calculation is done recursively [11]:

$$
\begin{aligned}
& p\left(\Omega_{j}^{k} \mid z_{k}\right) \quad=\quad p\left(\Psi_{j}(k), \Omega_{p(j)}^{k-1} \mid z_{k}\right) \\
& \underset{\text { Markov }}{\stackrel{\text { Bayes+ }}{=}} \eta p\left(z_{k} \mid \Psi_{j}(k), \Omega_{p(j)}^{k-1}\right) p\left(\Psi_{j}(k) \mid \Omega_{p(j)}^{k-1}\right) \text {. } \\
& p\left(\Omega_{p(j)}^{k-1}\right) .
\end{aligned}
$$

The rightmost term on the right-hand side is the recursive term, i.e., the probability of its parent. Factor $\eta$ is a normalizer. The leftmost term on the right-hand side after the normalizer $\eta$ is the measurement likelihood. We assume that a measurement $z_{k}$ associated with a door $j$ has a Gaussian pdf centered around the measurement prediction $\hat{z}_{k}^{j}$ with innovation covariance matrix $\mathcal{S}_{k}^{j}, \mathcal{N}\left(z_{k}\right):=\mathcal{N}\left(z_{k} ; \hat{z}_{k}^{j}, \mathcal{S}_{k}^{j}\right)$. Here, the innovation covariance matrix is the uncertainty of the door w.r.t. the current trajectory and is described in Section V. We further assume the pdf of a measurement belonging to a new door to be uniform in the observation volume $V$ with probability $V^{-1}$. Hence, we have

$$
p\left(z_{k} \mid \Psi_{j}(k), \Omega_{p(j)}^{k-1}\right)=\mathcal{N}\left(z_{k}\right)^{\delta} V^{\delta-1}
$$

with $\delta$ being 1 if and only if the measurement has been associated with an existing door, 0 otherwise. The central term on the right-hand side of Equation (1) is the probability of an assignment set, $p\left(\Psi_{j}(k) \mid \Omega_{p(j)}^{k-1}\right)$, which is composed of the following two terms: the probability of detection $p_{d e t_{j}^{k}}$ and the probability of a new door. In our case the probability of detection is equal to choosing one of the current candidate doors, i.e., all doors within an uncertainty ellipsoid. Therefore, $p_{\operatorname{det}_{j}^{k}}:=\mathrm{NC}\left(\mathbf{x}_{1: k}, \Omega_{p(j)}^{k-1}\right)^{-1}$, with $\mathrm{NC}\left(\mathbf{x}_{1: k}, \Omega_{p(j)}^{k-1}\right)$ being the number of door candidates, assuming the trajectory $\mathbf{x}_{1: k}$ within the world $\Omega_{p(j)}^{k-1}$. Assuming the number of new doors following a Poisson distribution with expected number of doors $\lambda_{n e w} V$ in the observation Volume $V$ we obtain

$$
p\left(\Psi_{j}(k) \mid \Omega_{p(j)}^{k-1}\right)=p_{\text {det }_{j}^{k}}^{\delta} \cdot \mu\left(1-\delta ; \lambda_{\text {new }} V\right)
$$


Time:

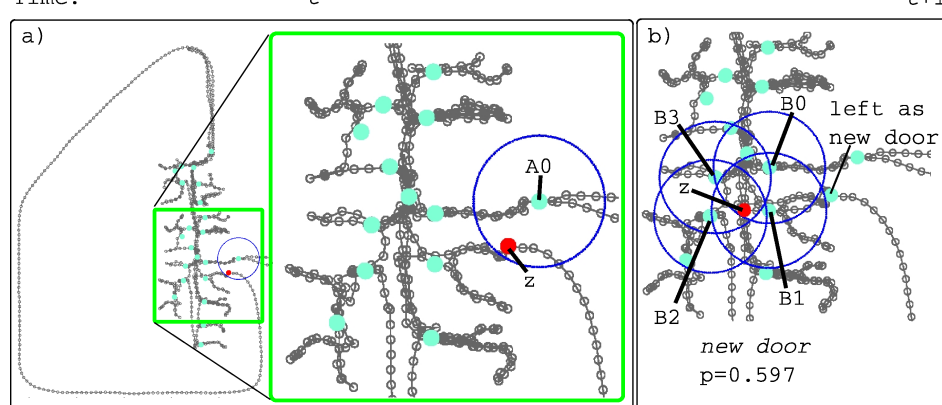

$t+1$

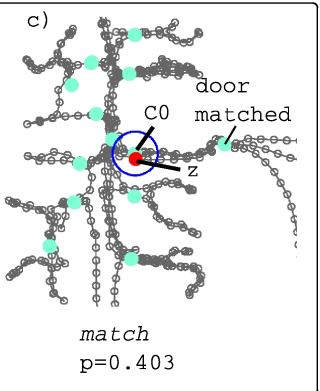

t $\quad t+1$

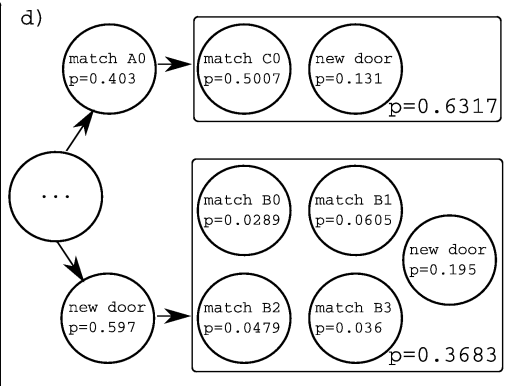

Fig. 3. A snapshot from our experiment which is described in detail in Section V. (a) The human re-enters the building through door A0. Based on the MHT decision new door and match with AO different hypothesis are generated (b) and (c). The probabilities of the hypothesis are depicted in (d).

where $\mu(n ; \lambda V):=\frac{(\lambda V)^{n} \exp (\lambda V)}{n !}$ is the Poisson distribution for $\mathrm{n}$ events given the average rate of events is $\lambda V$. Therefore, Equation (1) can be reformulated into

$$
\begin{aligned}
& p\left(\Omega_{j}^{k} \mid z_{k}\right)=p\left(\Psi_{j}(k), \Omega_{p(j)}^{k-1} \mid z_{k}\right) \\
& \begin{array}{l}
\text { Bayes+ } \\
\underset{\text { Markov }}{=}
\end{array} \quad \eta p\left(z_{k} \mid \Psi_{j}(k), \Omega_{p(j)}^{k-1}\right) p\left(\Psi_{j}(k) \mid \Omega_{p(j)}^{k-1}\right) \text {. } \\
& \text { Markov } p\left(\Omega_{p(j)}^{k-1}\right) \\
& =\eta \mathcal{N}\left(z_{k}\right)^{\delta} V^{\delta-1} p_{\operatorname{det}_{j}^{k}}^{\delta}\left(\lambda_{n e w} V\right)^{1-\delta} . \\
& \exp \left(\lambda_{\text {new }} V\right)(1-\delta) !^{-1} p\left(\Omega_{p(j)}^{k-1}\right) .
\end{aligned}
$$

Observing that $(1-\delta)$ ! is always 1 (since $\delta$ is $\in\{0,1\}$ ) and noting that $\exp \left(\lambda_{\text {new }} V\right)$ can be taken into the normalizer $\eta$, we can finally rewrite Equation (4) into

$$
p\left(\Omega_{j}^{k} \mid z_{k}\right)=\eta\left(\mathcal{N}\left(z_{k}\right) p_{\operatorname{det}_{j}^{k}}\right)^{\delta} \cdot \lambda_{n e w}^{1-\delta} \cdot p\left(\Omega_{p(j)}^{k-1}\right) .
$$

Up to now, we can reliably detect doors and calculate the probability of a data association. In the next section we address the remaining questions during our simultaneous localization and mapping procedure, namely the detection of possible door candidates (i.e., loop closures), the calculation of the innovation covariance and the algorithms which were utilized in order to correct the trajectory.

\section{Simultaneous Localization And Mapping}

We address the simultaneous localization and mapping problem by its graph based formulation. A node in the graph represents a $3 \mathrm{DoF}$ pose of the human pose (i.e., the center of the hip) or the $3 \mathrm{DoF}$ pose of a door and an edge between two nodes models a spatial constraint between them. These spatial constraints arise either from incremental odometry or by detecting a previously observed door (i.e., by opening/closing it). In our case, the edges are labeled with the relative motion between two nodes. To compute the spatial configuration of the nodes which best satisfy the constraints encoded in the edges of the graph, we utilize stochastic gradient descent optimization [4]. Performing this optimization whenever a door has been detected allows us to reduce the uncertainty in the current pose estimate.

Since we are only able to detect the fact that there is a door, we have to track different possibilities of data association, namely whether the current detected door is one of the already mapped doors, or whether the door has not been perceived before. We therefore utilize multi hypothesis tracking as described in the previous section for all possible outcomes. To detect a potential loop closure (i.e., recognize a previously seen door), we identify all former doors which are within the uncertainty ellipsoid of the current pose by a Dijkstra projection of the node covariances starting from the current position. The innovation covariance is directly used for calculating the likelihood of the door as described in Equation (5). All doors being within the $99.9 \%$ confidence region of the current pose are considered as potential loop closure candidates, and together with the possibility of the current detected door being a new door, give raise to $n+1$ different outcomes, given the number of loop closure candidates is $n$. For each of these association possibilities we create a separate graph, encode the selected constraint and optimize it. The multi hypothesis tree therefore grows exponentially in time and pruning of this tree is mandatory in order to keep computational costs reasonable. In our case, we utilize $N$-scan-back pruning as proposed by Cox and Hingorani [3], which works as follows: The N-scan-back algorithm considers an ancestor hypothesis at time $k-N$ and looks ahead in time to all its children at the current time $k$ (the leaf nodes). The probabilities of the children are summed up and propagated to the parent node at time $k-N$. Given the probabilities of the possible outcomes at time $k-N$, the branch with the highest probability at time $k$ is maintained whereas all others are discarded. Since in our case, a step in the MHT only arises when a door has been detected, this is identical to localize $N$ steps ahead in time (at door level). An example of this approach is visualized in Figure 3. This example shows a snapshot of one experiment which is described in detail in Section VI. At the specific time $t$, the human walked around the builing leaving at the top exit and entered the building through the main entry labeled A0 in 3(a). Starting from the pose $z$, where the current door was detected, the uncertainty of the pose was back-propagated utilizing Dijkstra expansion. Since we used the same uncertainty for $x$ and $y$, the resulting ellipsoid is a circle. Note that due to the back-propagation of the uncertainty the current pose is in the uncertainty region of the door A0. For better visibility, only the doors being considered as candidates are shown with their uncertainty regions. Therefore, only two data associations are possible in this case, namely matching the current door with $\mathrm{A} 0$, 

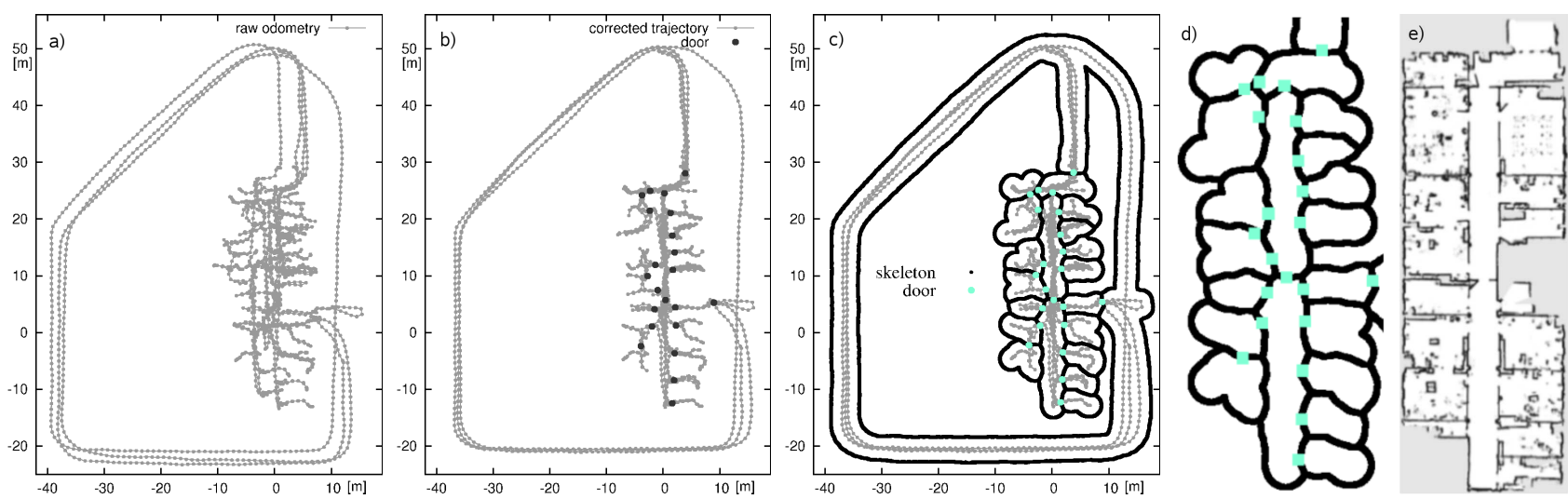

Fig. 4. The first experiment contains a trajectory of about $1.6 \mathrm{~km}$. The raw odometry is depicted in (a), whereas the most likely trajectory based on the detected doors is visualized in (b). Based on the corrected trajectory and the knowledge about doors, a topological map of the environment can be generated. A skeleton of the environment is generated afterwards by enlarging the free space around the trajectory up to a maximum (c). A close-up view of the internal part is also shown in (d). A laser map of the same enviornment is depcited in (e). A close up view of the trajectory is also shown in Figure 1.

which in this case is the correct association, or marking it as a new door. Calculating the posterior probability of each association leads to $p=0.597$ for the case new door and $p=0.403$ for the correct association. A maximum likelihood approach therefore selects the wrong association. However, as the human enters the building and opens another door, given the previous association, different possible outcomes are possible. Figure 3(b) depicts the situation for the case that the previous decision was new door and Figure 3(c) shows the situation for the decision match with AO. Given this sequence of doors, the full posterior of the branch match with $A O$ at time $t$ sums up to 0.6317 while the probability for the branch for new door sum up to 0.3683 (see Figure 3(d)). Here, a N-scan-back of 2 would be sufficient to keep track of the correct data association, since the MHT would decide to keep match with $A O$ at time $t$ and discard the other branch.

The output of this approach can be used to generate an approximate map of the environment. Assuming that doors separate rooms, we can cut the trajectory based on the locations of individual doors. Each segment now contains all points belonging to one room only. Given the orientation of a door we can merge subsequent segments which are both connected to the same door and on the same side. In order to seek for walls, we can furthermore enlarge the trajectory until it touches a trajectory belonging to another room or up to a threshold $d$, which was set to $2.5 \mathrm{~m}$ in all our experiments. An outcome of this process is shown in Figure 4(c).

\section{EXPERIMENTS}

We evaluated the approach described above on different data sets utilizing the motion of two humans including walking inside and outside of various buildings. Videos of each experiments can be found on the web (http://ais.informatik.uni-freiburg.de/projects/mvn). They show the incremental update of the final best hypothesis. Our current system, though not optimized, is able to perform an incremental update at a rate of $10 \mathrm{~Hz}$ on a state-of-the-art desktop computer. The first experiment contains a trajectory of approximately $1.6 \mathrm{~km}$ including 133 door actions and is depicted in Figure 4. Given the learned motion templates, we were able to detect 125 out of the
133 doors. The pure odometry is shown in Figure 4(a). In this experiment, the raw odometry is already good, since we intentionally omitted walking around tables and chairs which would result in high pose errors due to magnetic disturbances. Therefore, a variance of $0.03 \mathrm{~m}$ per meter and a $\mathrm{N}$-scan-back of 7 were sufficient to correct the odometry. We have chosen $\lambda_{\text {new }}=0.04$ since this value is approximately obtained by dividing the number of doors by the area covered through the doors. Note, that although $\lambda_{n e w}$ is dependant on the building the human is operating in, small changes will not alter the final outcome. However, if operating in a hotel, $\lambda_{n e w}$ should be siginifcantly higher than if operating in a warehouse. Based on the detection and tracking of individual doors, the map was corrected as depicted in Figure 4(b). Note that we show the maximum likelihood map of the multi hypothesis tracking only. Given the free space traversed by the human and the knowledge that doors separate rooms, we can enlarge the current trajectory up to a threshold $d=2.5 \mathrm{~m}$ to seek for walls, i.e., build a Voronoi diagram, based on all poses within a room (see Figure 4(c)). The resulting map of the inner part is depicted in Figure 4(d). For comparison we enlarged the indoor part of this experiment and compared it to a laser map, which is shown in Figure 4(e) and Figure 1.

The second experiment contains a trajectory of approximately $1.3 \mathrm{~km}$ and was obtained by walking inside a university building containing several seminar rooms. Here, we intentionally walked closely around rows of tables and chairs. The magnetic disturbances led to a high pose error, as can be seen in the raw odometry (see Figure 5(a)). We therefore used a high variance of $0.2 \mathrm{~m}$ per meter to make sure that the variance is not over-confident. Although the initial odometry differs up to $30 \mathrm{~m}$ for the same place, we were able to correct it as shown in Figure 5(b). The map obtained by our approach is visualized in Figure 5(c) and a floor plan of the same building is depicted in (d). In this experiment we detected all 63 doors up to an accuracy of 0.5 seconds wrt. a manually labeled ground truth.

The third experiment contains a trajectory of a person who is about $20 \mathrm{~cm}$ taller than the person whose motions were used for training the templates. The parameters used to correct this trajectory were the same as for the second 

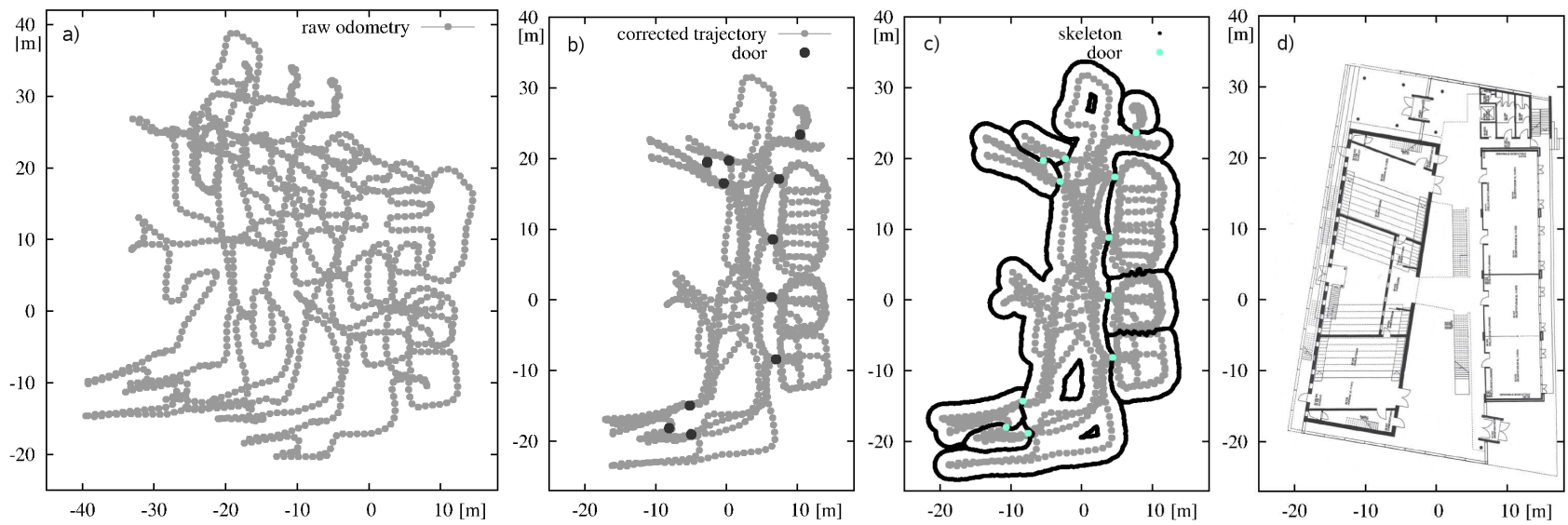

Fig. 5. The second experiment containing a trajectory of about $1.3 \mathrm{~km}$ : the raw trajectory is depicted in (a). We intentionally walked closely around tables and chairs resulting in a high pose error due to magnetic disturbances. The corrected trajectory using our approach is depicted in (b). The approximate map of the environment and a floor plan of the building are shown in (c) and (d) respectively.
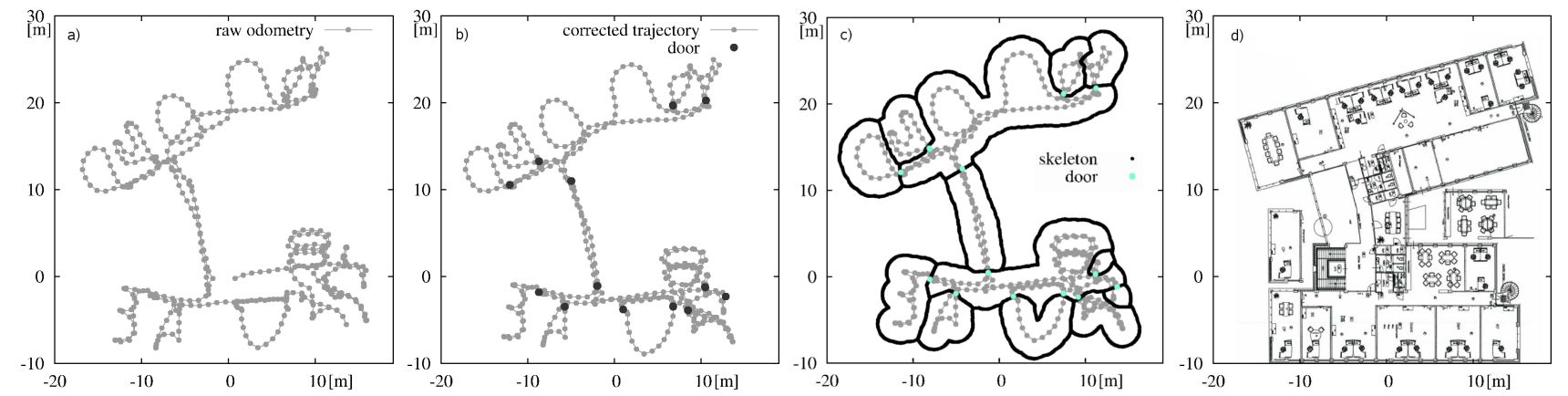

Fig. 6. The third experiment containing the motion from a different person. The raw odometry and the corrected trajectory based on our approach are depicted in (a) and (b) respectively. The calculated approximate map is shown in (c) and a floor plan of the same building is depicted in (d).

experiment. The outcome of this experiment is shown in Figure 6. Here, 24 out of 27 doors were detected.

\section{CONCLUSIONS}

In this paper, we presented a novel approach for approximate mapping of indoor environments using sensed human motion. Our approach considers the trajectory of the person as motion constraints and door handling events detected using specific motion templates as landmarks within a graphbased SLAM approach. To cope with the high data association uncertainty, we employ a multi-hypothesis tracking approach. Our approach has been implemented and tested on real data acquired by people walking inside and outside of various buildings. The experimental results demonstrate that our approach is able to robustly keep track of the true data association and accurately estimate the trajectory taken by the person. Additionally, we can create approximate maps of the environment which accurately resemble the true layouts.

\section{ACKNOWLEDGMENTS}

The authors would like to thank Gian Diego Tipaldi for the fruitful discussions. We would also like to thank Xsens for providing us with the additional dataset used in the third experiment. This work has been supported by the EC under contract number FP6-IST-034120 Micro/Nano based Systems

\section{REFERENCES}

[1] S. S. Bao, L. Intille. Activity recognition from user-annotated acceleration data. pages 1-17. Springer, 2004.
[2] B. Cinaz and H. Kenn. HeadSLAM - simultaneous localization and mapping with head-mounted inertial and laser range sensors. Wearable Computers, IEEE International Symposium, 0:3-10, 2008.

[3] Ingemar J. Cox and Sunita L. Hingorani. An efficient implementation of Reid's multiple hypothesis tracking algorithm and its evaluation for the purpose of visual tracking. IEEE Transactions on Pattern Analysis and Machine Intelligence, 18(2):138-150, 1996.

[4] G. Grisetti, D. Lodi Rizzini, C. Stachniss, E. Olson, and W. Burgard. Online constraint network optimization for efficient maximum likelihood mapping. In Proc. of the IEEE Int. Conf. on Robotics \& Automation (ICRA), Pasadena, CA, USA, 2008.

[5] G. Grisetti, C. Stachniss, and W. Burgard. Improving grid-based slam with Rao-Blackwellized particle filters by adaptive proposals and selective resampling. In Proc. of the IEEE Int. Conf. on Robotics \& Automation (ICRA), pages 2443-2448, Barcelona, Spain, 2005.

[6] S.-W. Lee and K. Mase. Activity and location recognition using wearable sensors. IEEE Pervasive Computing, 1(3):24-32, 2002.

[7] H. Liu, H. Darabi, P. Banerjee, and J. Liu. Survey of wireless indoor positioning techniques and systems. Systems, Man and Cybernetics, Part C: Applications and Reviews, IEEE Transactions on, 37(6):10671080, 2007.

[8] M. Müller and T. Röder. Motion templates for automatic classification and retrieval of motion capture data. In Proceedings of the 2006 ACM SIGGRAPH/Eurographics symposium on Computer animation, pages 137-146. Eurographics Association Aire-la-Ville, Switzerland, Switzerland, 2006.

[9] L. Rabiner and B.H. Juang. Fundamentals of speech recognition. 1993.

[10] N. Ravi, N. Dandekar, P. Mysore, and M. L. Littman. Activity recognition from accelerometer data. In In Proceedings of the Seventeenth Conference on Innovative Applications of Artificial Intelligence(IAAI), pages 1541-1546. AAAI Press, 2005.

[11] D. Reid. An algorithm for tracking multiple targets. IEEE Transactions on Automatic Control, 24:843-854, Dec 1979.

[12] G. Schindler, C. Metzger, and T. Starner. A wearable interface for topological mapping and localization in indoor environments. In Mike Hazas, John Krumm, and Thomas Strang, editors, LoCA, volume 3987 of Lecture Notes in Computer Science, pages 64-73. Springer, 2006. 